\title{
Protective effects of geniposide and ginsenoside Rg1 combination treatment on rats following cerebral ischemia are mediated via microglial microRNA-155-5p inhibition
}

\author{
JUN WANG ${ }^{1}$, DAN LI ${ }^{2}$, JINCAI HOU ${ }^{2}$ and HONGTAO LEI ${ }^{3}$ \\ ${ }^{1}$ Institute of Basic Theory, China Academy of Chinese Medical Sciences, Beijing 100700; \\ ${ }^{2}$ Jingjinji United Innovation Pharmaceutical Research Company, Beijing 100083; \\ ${ }^{3}$ Experimental Research Center, China Academy of Chinese Medical Sciences, Beijing 100700, P.R. China
}

Received June 7, 2017; Accepted November 2, 2017

DOI: $10.3892 / \mathrm{mmr} .2017 .8221$

\begin{abstract}
Geniposide, an active component of Gardenia, has been reported to protect against cerebral ischemia in animals. Ginsenoside Rg1, a component of Panax notoginseng, is usually administered in combination with Gardenia for the treatment of acute ischemic stroke; however, there are unknown effects of ginsenoside $\mathrm{Rg} 1$ that require further investigation. In the present study, the effects of geniposide and ginsensoide $\mathrm{Rg} 1$ combination treatment on focal cerebral ischemic stroke were investigated. For in vivo analysis, male rats were separated into three groups, including the (control), model and geniposide + ginsenoside $\operatorname{Rg} 1$ groups $(n=8$ per group). A middle cerebral artery occlusion model was established as the model group. The treatment group was treated with geniposide $(30 \mathrm{mg} / \mathrm{kg}$, tail vein injection $)+$ ginsenoside $\mathrm{Rg} 1$ (6 mg/kg,tail vein injection), and the model group received saline instead. Neurobehavioral deficits, infarct volume, brain edema, and the expression of microRNA (miR)-155-5p and $\mathrm{CD} 11 \mathrm{~b}$ by reverse transcription-quantitative polymerase chain reaction (RT-qPCR) and immunohistochemistry, were assessed following $24 \mathrm{~h}$ of ischemia. For in vitro analysis, BV2 mouse microglial cells were cultured and exposed to geniposide $(40 \mu \mathrm{g} / \mathrm{ml})+$ ginsenoside $\operatorname{Rg} 1(8 \mu \mathrm{g} / \mathrm{ml})$ during various durations of oxygen-glucose deprivation (OGD). The expression levels of miR-155-5p, pri-miR-155 and pre-miR-155 were detected by RT-qPCR. The results demonstrated that increases in brain infarct volume, edema volume, CD11b-positive cells and miR-155-5p levels were alleviated following geniposide + ginsenoside administration in rats exposed to ischemia. Furthermore, geniposide + ginsenoside
\end{abstract}

Correspondence to: Dr Jun Wang, Institute of Basic Theory, China Academy of Chinese Medical Sciences, 16 Dong Zhi Men Nei Nan Xiao Jie, Beijing 100700, P.R. China

E-mail: maggie.wj@126.com

Key words: geniposide, ginsenoside Rg1, microRNA-155, microglia, brain ischemia
Rg1 treatment suppressed the miR-155-5p, pri-miR-155 and pre-miR-155 expression levels in OGD-injured BV2 microglial cells. The results of the present study demonstrated that tail vein administration of geniposide in combination with ginsenoside $\operatorname{Rg} 1$ protected against focal cerebral ischemia in rats through inhibition of microglial miR-155-5p following ischemic injury, which may serve as a novel therapeutic agent for the treatment of strokes.

\section{Introduction}

Ischemic stroke caused by blood vessel occlusion is a major cause of morbidity and mortality in humans. Numerous pathological processes are associated with ischemic neuronal damage, including inflammation, energy metabolism disturbance, necrotic and apoptotic cell death, and excitotoxicity (1). Previous studies have aimed to improve the understanding of the underlying mechanisms of ischemic stroke; however, further investigation is required to identify a potential target for the effective prevention and treatment of stroke. Recent clinical and experimental studies have demonstrated that alternative complementary medicine may exhibit a unique efficacy in the treatment of stroke $(2,3)$.

MicroRNA (miRNA/miR), which are non-coding RNAs, are frequently employed in research and are characterized by their regulatory effects on gene expression by inhibiting mRNA translation and/or mediating mRNA degradation $(4,5)$. As numerous pathways are implicated in cerebral ischemic injury, including oxidative stress, excitotoxicity, mitochondrial dysfunction and inflammation, miRNAs have been associated with cerebral ischemic injury due to their roles in ischemic processes, including neuronal cell differentiation $(6,7)$, dendritic plasticity and neuronal outgrowth modulation (8). As a multifunctional miRNA, miR-155 expression is affected by cerebral ischemia (9-11) and has been reported to be involved in the regulation of a variety of physiological and pathological processes, including inflammation and immunity $(12,13)$. Several inflammatory and cytotoxic factors, including tumor necrosis factor- $\alpha$, interleukin (IL)-1 $\beta$ and IL-6, secreted by activated microglia may exert an effect on neuronal cell survival (14). miR-155 
may regulate the immune response mediated by microglia in various pathological states (15-17).

Gardenia is the fruit of Gardenia jasminoides Ellis (Rubiaceae), which is a commonly used Chinese herb. Gardenia has been reported to exhibit therapeutic effects on hepatic disorders, brain diseases, inflammatory diseases and contusions (18-20). A previous study reported that geniposide, an active component of Gardenia, reduces neuronal cell death and increases cell viability following oxygen and glucose deprivation (OGD) (21). Our previous study also revealed that geniposide exhibits protective effects against infarction volume, microglial activation and inflammatory cytokine release in middle cerebral artery occlusion (MCAO) model rats (22). In clinical and experimental research, Gardenia has previously been used in combination with Panax notoginseng to treat brain diseases, including cerebrovascular disease and Alzheimer's disease $(23,24)$. For rats injured by local ischemia, ginsenoside $\mathrm{Rg} 1$, a component of Panax notoginseng, was reported to alleviate neurological function deficits, as determined by improvements in the learning and memory abilities, in addition to a reduction in the apoptosis of neurons in the hippocampus CA1 zone $(25,26)$. The present study aimed to investigate whether tail vein-administered geniposide in combination with ginsenoside $\operatorname{Rg} 1$ may protect against ischemic stroke via miR-155 inhibition. An in vivo investigation into miR-155-5p expression, and the effects of geniposide + ginsenoside $\mathrm{Rg} 1$ on miR-155-5p expression within the brain and activation of microglia, as determined by immunohistochemistry for $\mathrm{CD} 11 \mathrm{~b}$, was performed. In addition, an in vitro study was performed to analyze the effects of geniposide + ginsenoside Rg1 on miR-155-5p expression in BV2 microglial cells.

\section{Materials and methods}

Animals and grouping. Specific pathogen and virus antibody-free grade male Sprague-Dawley rats $(n=56 ; 253.4 \pm 20.3 \mathrm{~g}$; 7-8 weeks-old) were obtained from Beijing Vital River Laboratory Animal Technology Co., Ltd. (Beijing, China). In accordance with the UK Animals (Scientific Procedures) Act of 1986 (27), the animal experiments were performed with the approval of the ethics committee of the Institute of Basic Theory, China Academy of Chinese Medical Sciences (Beijing, China). The rats were housed in rooms maintained at $23 \pm 2^{\circ} \mathrm{C}$ in a $12 \mathrm{~h}$ light/dark cycle and fed a rodent standard diet with free access to water. The animals were randomly divided into three groups ( $\mathrm{n}=8$ per group): Sham, model and geniposide $(30 \mathrm{mg} / \mathrm{kg})+$ ginsenoside Rg1 (6 mg/kg) groups. For the sham group, the rats underwent similar surgery to the model group, without nylon filament insertion; rats in the model group were administrated saline via the tail vein immediately following MCAO. Rats in the geniposide + ginsenoside Rg1 group were administered geniposide $(30 \mathrm{mg} / \mathrm{kg})+$ ginsenoside $\mathrm{Rg} 1(6 \mathrm{mg} / \mathrm{kg})$ via the tail vein immediately following MCAO. Geniposide (purity, $>95 \%$; cat. no. 110749-201718) and ginsenoside Rg1 (purity, >95\%; cat. no. 110703-201731) were purchased from the National Institutes for Food and Drug Control (Beijing, China).

MCAO model establishment. The MCAO model was established as described by Longa et al (28). Rats were anesthetized with $10 \%$ chloral hydrate $(350 \mathrm{mg} / \mathrm{kg}$ body weight, intraperitoneal injection) $(29,30)$. Subsequently, a midline incision was made to expose the left common carotid artery and the external carotid artery (ECA), including the occipital artery branches. Following this, blood from the occipital artery and superior thyroid artery branches of the ECA was coagulated using a bipolar electrocoagulator. The internal carotid artery (ICA) was isolated and carefully separated from the adjacent vagus nerve. Following ICA exposure, the origin of the left middle cerebral artery was occluded using a $0.25 \mathrm{~mm}$ diameter nylon filament coated with poly-L-lysine, which was inserted into the ICA via the ECA. Rectal temperature was maintained at $37^{\circ} \mathrm{C}$ during and following surgery with a temperature-controlled heating pad (CMA 150 Carnegie Medicin AB, Solna, Sweden). After 2, 6 and 24 h, the ischemic procedure was complete.

Infarct size and brain edema detection. Rats of the $24 \mathrm{~h}$ ischemia group were selected for in vivo analysis; statistical calculations demonstrated no significant difference when comparing the 2 and $6 \mathrm{~h}$ ischemia group rats with the control group. Following the performance of ischemic injury for different time periods, all animals were sacrificed. Brain tissues were removed and prepared into $2 \mathrm{~mm}$ coronal sections. The sections were stained with $1.5 \%$ 2,3,5-triphenyltetrazolium chloride at $37^{\circ} \mathrm{C}$ for $10 \mathrm{~min}$ and fixed in $4 \%$ paraformaldehyde for $24 \mathrm{~h}$ at room temperature. The images of the stained slices were analyzed using Image-Pro Plus 6.0 (Media Cybernetics, Inc., Rockville, MD, USA) to determine the infarct size, which was expressed as the percentage of the whole brain tissue. Brain edema was calculated according to the following equation: [(volume of ipsilateral hemisphere-volume of contralateral hemisphere)/volume of contralateral hemisphere] x100. The experiment was independently repeated three times.

Cell culture and in vitro model. Mouse BV2 microglial cells were purchased from the Institute of Basic Medical Sciences of the China Science Academy (Beijing, China) and cultured in Dulbecco's modified Eagle medium/Ham's F12 (DMEM/F12; Gibco; Thermo Fisher Scientific, Inc., Waltham, MA, USA) culture medium containing $10 \%$ fetal bovine serum (Thermo Fisher Scientific, Inc.) and 1\% penicillin and streptomycin (Sigma-Aldrich; Merck KGaA, Darmstadt, Germany) in a humidified $5 \% \quad \mathrm{CO}_{2} / 95 \%$ air atmosphere at $37^{\circ} \mathrm{C}$. The microglial cells were passaged at $90 \%$ confluence, with two to three passages per week. To mimic the OGD pathological process, glucose-free DMEM (Gibco; Thermo Fisher Scientific, Inc.) was used instead of the culture medium and oxygen was restricted in the flasks by keeping the culture flasks in a sealed tank with a persistent low-flow $(1.5 \mathrm{l} / \mathrm{min})$ of $95 \% \mathrm{~N}_{2}$ and $5 \% \mathrm{CO}_{2}$ mixture. The inlet and outlet ends of the tubes were then clipped and the sealed tank was returned to the incubator. DMEM was used to dissolve geniposide and ginsenoside $\mathrm{Rg} 1$ and prepare the storage solution. A total of $40 \mu \mathrm{g} / \mathrm{ml}$ geniposide $+8 \mu \mathrm{g} / \mathrm{ml}$ ginsenoside $\mathrm{Rg} 1$ was dissolved within the culture media. After 4,8 and $16 \mathrm{~h}$ of OGD exposure at $37^{\circ} \mathrm{C}$, with or without geniposide $(40 \mu \mathrm{g} / \mathrm{ml})+$ ginsenoside $\mathrm{Rg} 1(8 \mu \mathrm{g} / \mathrm{ml}), \mathrm{BV} 2$ cells were collected for the analysis of miR-155-5p expression. The experiment was independently repeated three times. 
miR-155-5p, pri-miR-155 and pre-miR-155 quantification by reverse transcription-quantitative polymerase chain reaction ( $R T-q P C R)$. Total RNA of brain tissue and cultured BV2 microglial cells was isolated and analyzed using a Reverse Transcriptase kit (Suzhou GenePharma, Co., Ltd., Suzhou, China). The temperature and duration of reverse transcription was performed at $30^{\circ} \mathrm{C}$ for $10 \mathrm{~min}, 42^{\circ} \mathrm{C}$ for $30 \mathrm{~min}$ and $99^{\circ} \mathrm{C}$ for $5 \mathrm{~min}$. A stem-loop real-time PCR system was used to determine the level of miRNAs using the Maxima SYBR Green qPCR Master Mix (Fermentas; Thermo Fisher Scientific, Inc., Waltham MA, USA) and a StepOne Sequence Detector (Applied Biosystems; Thermo Fisher Scientific, Inc.). qPCR was performed at $95^{\circ} \mathrm{C}$ for $10 \mathrm{~min}$ followed by 40 cycles at $95^{\circ} \mathrm{C}$ for $15 \mathrm{sec}$ and $60^{\circ} \mathrm{C}$ for $60 \mathrm{sec}$. The following primer sequences were used: miR-155 forward, 5'-GCTTCGGTTAATGCTAATCGTG-3'; miR-155 reverse, 5'-AGAGCAGGGTCCGAGGTA-3'; U6 forward, 5'-ATTGGA ACGATACAGAGAAGATT-3'; U6 reverse, 5'-GGAACGCTTCACGAATTTG-3'; pre-miR-155 forward, 5'-TGCTAATTGTGATAGGGGTTTT-3'; pre-miR-155 reverse, 5'-TAT GGT TGT TCACGACTCCTTCAC-3'; pri-miR-155 forward, 5'-AGGCTTTTCCTGGGCACC-3'; and pri-miR-155 reverse, 5'-CATGAACAAACCACAACG AGC-3'. RT-qPCR was performed in duplicate and relative expression of miR-155-5p, pri-miR-155 and pre-miR-155 were normalized to U6 expression and determined by the $2^{-\Delta \Delta \mathrm{Cq}}$ method (31). The experiment was independently repeated three times.

Immunohistochemistry. Brain tissues from rats were removed for immunohistochemistry analysis at the termination of ischemia. Brain tissues were fixed in $4 \%$ paraformaldehyde overnight at room temperature. Sections $(5 \mu \mathrm{m})$ embedded in paraffin were deparaffinized in Histo-Clear ${ }^{\mathrm{TM}}$ (HS-200; National Diagnostics, Atlanta, GA, USA) rehydrated and washed with $0.01 \mathrm{M} \mathrm{PBS} ; 3 \%$ hydrogen peroxide in methanol was used at room temperature for $30 \mathrm{~min}$ to inhibit the endogenous peroxidase. Sections were incubated with rabbit anti-CD11b (1:500) antibody (cat. no. ab133357; Abcam, Cambridge, MA) at $4^{\circ} \mathrm{C}$ overnight following blocking of nonspecific antigens with goat serum (Gibco; Thermo Fisher Scientific, Inc.) at $37^{\circ} \mathrm{C}$ for $20 \mathrm{~min}$. Subsequently, sections were incubated with horseradish peroxidase-conjugated secondary antibodies at $37^{\circ} \mathrm{C}$ for $2 \mathrm{~h}$. Peroxidase detection was performed using a mixture containing $0.05 \%$ 3,3'-diaminobenzidine. Following staining, all sections were coverslipped and analyzed. The immunohistochemistry images were acquired using an Olympus Fluoview FV1000 microscope (LSI3-FV1000-Inverted; Olympus Corporation, Tokyo, Japan) and Image-Pro Plus 6.0 software (Media Cybernetics, Inc.). The experiment was independently repeated three times.

Statistical analysis. Data are presented as the mean + standard deviation. The statistical analysis was conducted with SPSS software (SPSS, Inc. Chicago, IL, USA). Significant differences among the groups were determined by one-way analysis of variance, followed by a Student-Newman-Keuls post-hoc test. $\mathrm{P}<0.05$ was considered to indicate a statistically significant difference.

\section{Results}

miR-155-5p expression in rats. In the present study, a rat MCAO model was established. miR-155-5p expression levels increased in a time-dependent manner; miR-155-5p expression levels were significantly increased in the ipsilateral brain tissue of rats in the $24 \mathrm{~h}$ ischemia group compared with the sham group $(\mathrm{P}<0.01$; Fig. 1A). The Cq values of 2, 6 and $24 \mathrm{~h}$ ischemia groups increased by 1.42-, 1.62- and 2.94-fold, respectively, compared with the sham group (Fig. 1A). To investigate whether the increase of miR-155-5p in the brain was region specific, miR-155-5p expression levels were observed within various brain regions at $24 \mathrm{~h}$ post-ischemia. The results demonstrated that miR-155-5p expression was significantly increased in the cortex and basal ganglia of the ipsilateral brain in MCAO models, compared with in the sham group $(\mathrm{P}<0.05$; Fig. $1 \mathrm{~B}$ and $\mathrm{C})$. However, a significant increase was not observed in the expression levels of miR-155-5p within the hippocampus of MCAO model rats, compared with in the sham group (Fig. 1D). Treatment with geniposide + ginsenoside Rg1 attenuated the miR-155-5p Cq value markedly compared with the model group $(1.67 \pm 0.52$ and $2.93 \pm 0.87$, respectively; $\mathrm{P}<0.05$; Fig. 1E). However, geniposide + ginsenoside $\mathrm{Rg} 1$ had no effect on the miR-155-5p expression levels in the basal ganglia of MCAO model rats (Fig. 1F).

Geniposide + ginsenoside Rg1 protects brain tissue against ischemic injury. To assess the in vivo protective effect of geniposide + ginsenoside Rg1 against ischemic injury, the percentage of cerebral infarction and edema was investigated within an MCAO rat model. Infarction was not observed in the sham group. Cerebral infarction within the model group was observed primarily in the frontal and parietal lobe of the cortex, the caudate nucleus and posterior limbs of the internal capsule (Fig. 2A). The results revealed that geniposide + ginsenoside Rg1 treatment significantly reduced brain infarction size and edema volume compared with the model group $(\mathrm{P}<0.05)$. In particular, the percentage of cerebral infarction and brain edema of rats subjected to geniposide + ginsenoside Rg1 was decreased by 34.2 and $42.6 \%$, respectively, compared with the MCAO model group (Fig. 2B and C).

Geniposide + ginsenoside Rg1 inhibits microglia stimulation. Immunohistochemistry demonstrated low levels of CD11b expression within the sham group (Fig. 3A). However, after $24 \mathrm{~h}$ of ischemia, CD11b immunoreactivity was visibly increased in the model group (Fig. 3B), which was primarily present in the cortex region, indicating a marked activation of microglial cells within ischemic penumbra. The positive staining of CD11b was primarily located within the cell membranes, while the microglial morphology altered from branch- to amoeba-like, indicating a marked stimulation. Following treatment with geniposide + ginsenoside $\mathrm{Rg} 1$, the expression of CD11b was suppressed in the brain parenchyma, compared with the model group (Fig. 3C), as indicated by a reduced number of CD11b-positive cells in the ischemic penumbra region. Semi-quantitative analysis revealed the relative gray values of immunohistochemical staining (Fig. 3D), which indicated that the number of CD11b-positive cells was markedly reduced by geniposide + ginsenoside $\mathrm{Rg} 1$ treatment in MCAO model rats $(\mathrm{P}<0.05)$. 
A
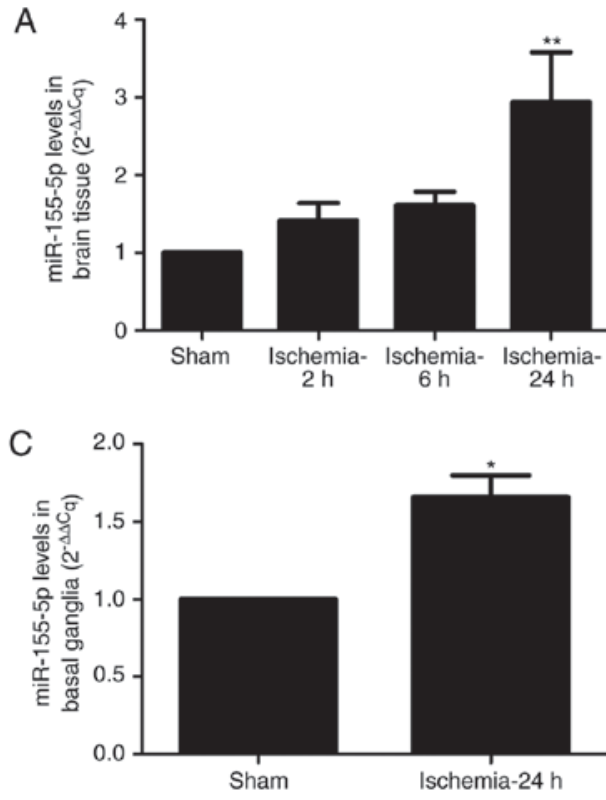

E

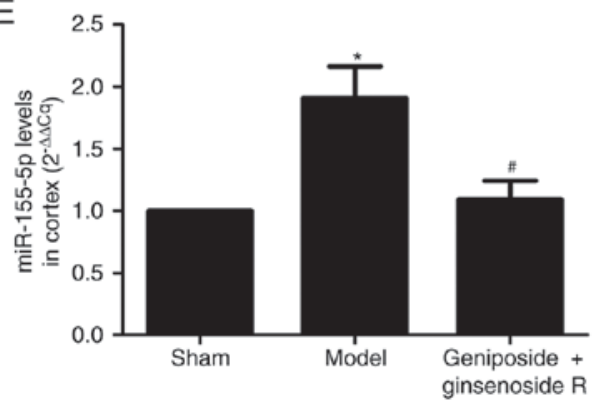

B

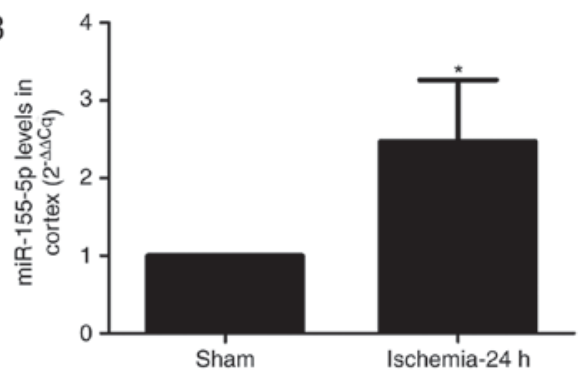

D

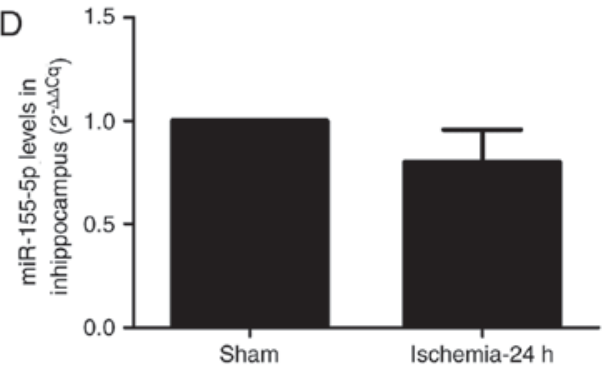

$\mathrm{F}$

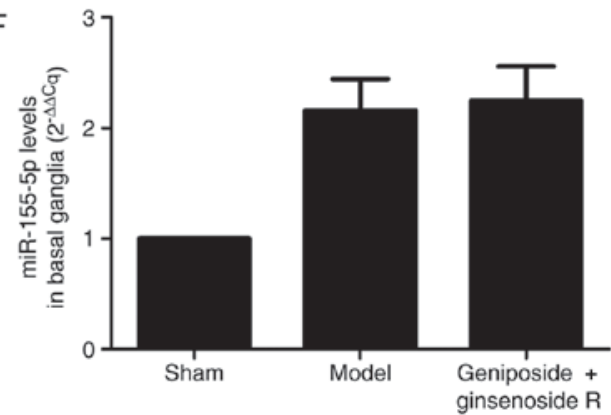

Figure 1. miR-155-5p expression levels are increased in MCAO model rats. (A) miR-155-5p expression levels in the ipsilateral brain tissue of MCAO rats increased in a time-dependent manner; ischemia-2, -6 and $-24 \mathrm{~h}$ indicates rats subjected to MCAO for 2, 6 and $24 \mathrm{~h}$, respectively. Reverse transcription-quantitative polymerase chain reaction analysis of miR-155-5p expression within the (B) ipsilateral cortex, (C) basal ganglia and (D) hippocampus of sham and $24 \mathrm{~h}$ ischemia groups. (E) Geniposide + ginsenoside Rg1 inhibited miR-155-5p expression levels in the cortex of MCAO model rats. (F) Geniposide + ginsenoside Rg1 exhibited no effects on miR-155-5p expression levels in the basal ganglia of MCAO model rats. Values are presented as the mean + standard deviation, $\mathrm{n}=8$ rats per group. ${ }^{*} \mathrm{P}<0.05$ and ${ }^{* *} \mathrm{P}<0.01$ vs. sham group, ${ }^{\#} \mathrm{P}<0.05$ vs. model group. miR, microRNA; MCAO, middle cerebral artery occlusion; geniposide + ginsenoside $\mathrm{R}$, geniposide + ginsenoside $\mathrm{Rg}$.

miR-155-5p expression within BV2 microglial cells. To further investigate the protective effects of geniposide + ginsenoside $\mathrm{Rg} 1$ against ischemic injury in vitro, BV2 microglial cells were cultured and the levels of immature forms of miR-155 (pri-miR-155 and pre-miR-155) and mature miR-155-5p were observed by RT-qPCR. As presented in Fig. 4A, miR-155-5p expression levels within the OGD $4 \mathrm{~h}$ group were not markedly altered compared with in control group. However, when the duration of ischemic was extended to 8 or $16 \mathrm{~h}$, miR-155-5p expression levels within BV2 microglial cells were significantly increased compared with the control group $(\mathrm{P}<0.001)$. Consequently, OGD $8 \mathrm{~h}$ was selected as the duration for stimulation for subsequent analysis of BV2 cells. Treatment of cells with geniposide + ginsenoside $\mathrm{Rg} 1$ for 8 h significantly decreased the expression levels of the immature and mature forms of miR-155. In particular, miR-155-5p expression within BV2 microglial cells incubated with geniposide + ginsenoside Rg1 was reduced by $93.9 \%$ compared with the model group (Fig. 4B; $\mathrm{P}<0.01$ ). In addition, following treatment with geniposide + ginsenoside Rg1, the expression levels of pri-miR-155 and pre-miR-155, the immature forms of miR-155, were significantly inhibited by 97.9 and $97 \%$, respectively, compared with the model group ( $\mathrm{P}<0.01$; Fig. $4 \mathrm{C}$ and $\mathrm{D})$.

\section{Discussion}

Ischemic stroke leads to a series of complex, multipart-cascade events that contribute to irreversible brain injury and neurovascular network dysfunction within the ischemic core (32). Activation of microglia and astrocytes following stroke leads to enhanced release of proinflammatory factors, which may persist for several weeks (33). Although the immune responses mediated by microglia serve an important role in pathogen clearance and tissue regeneration, without treatment, the inflammatory state may worsen neuronal injury (16). miRNAs are a type of small non-coding RNA that regulate target gene expression and are highly conserved in various eukaryotes (34). Numerous miRNAs have been reported to be involved in the regulation of several inflammation phases and responses to cerebral ischemia (35-37). Of these miRNAs, miR-155 has been demonstrated to be associated with the regulation of various physiological and pathological processes, including inflammation, immunity, 
A

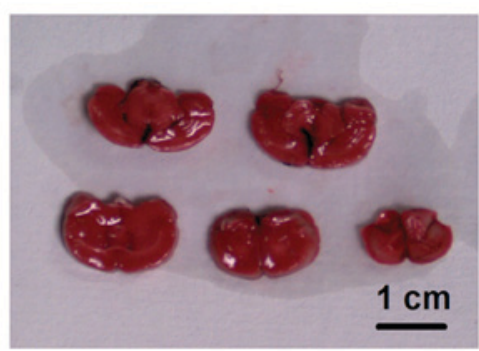

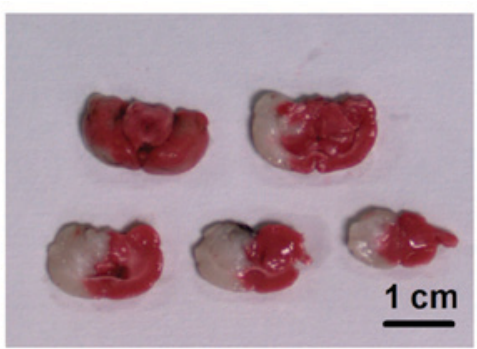

Model (ischemia $24 \mathrm{~h}$ )

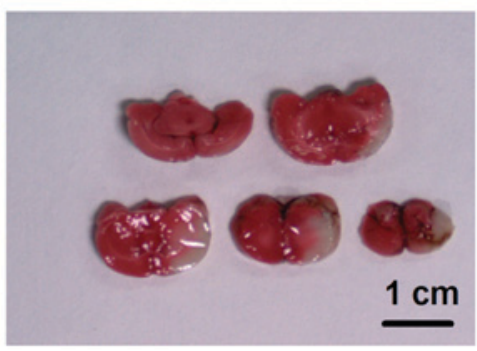

Geniposide + ginsenoside $\mathrm{R}$
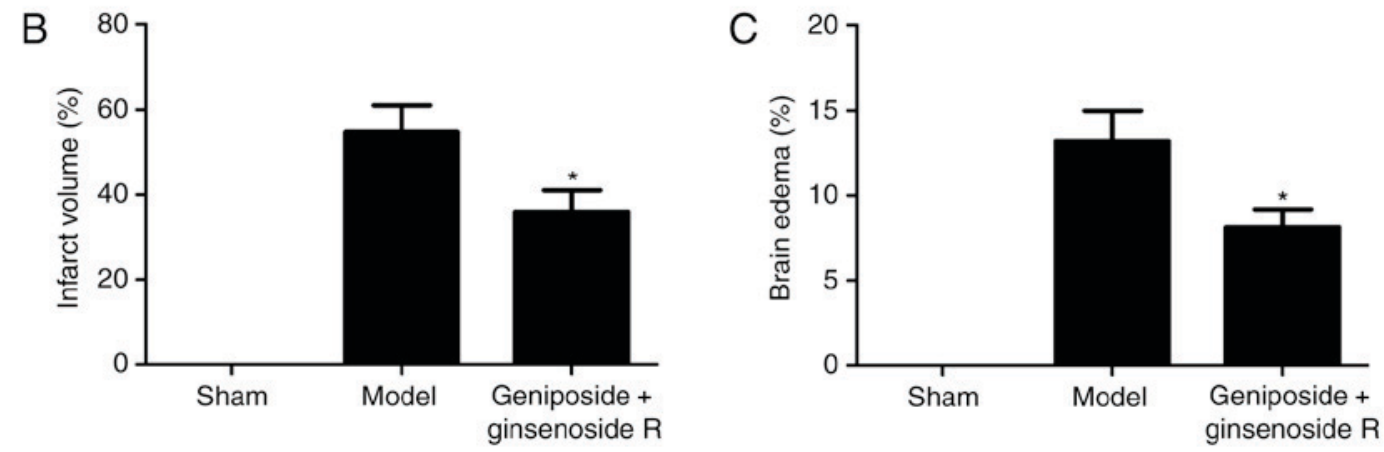

Figure 2. Treatment with geniposide + ginsenoside Rg1 attenuates brain infarction and brain edema following $24 \mathrm{~h}$ MCAO in rats. (A) Representative coronal sections stained by 2,3,5-triphenyltetrazolium. Effect of geniposide + ginsenoside Rg1 on (B) brain infarction volume and (C) brain edema in MCAO model rats. Values are presented as the mean + standard deviation, $\mathrm{n}=8$ rats per group. ${ }^{*} \mathrm{P}<0.05 \mathrm{vs}$. model group. MCAO, middle cerebral artery occlusion; geniposide + ginsenoside R, geniposide + ginsenoside Rg1.

A

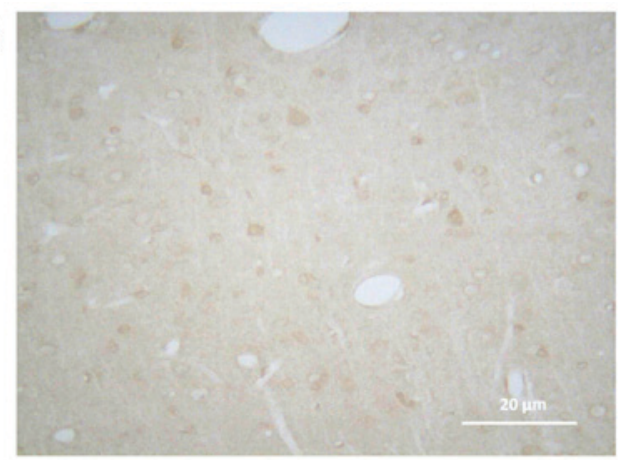

C

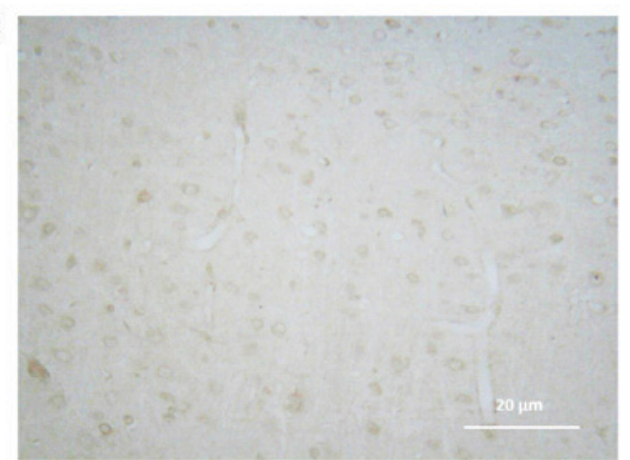

B

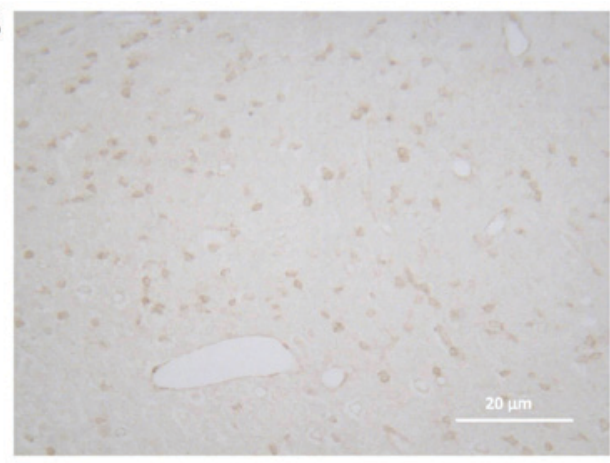

D

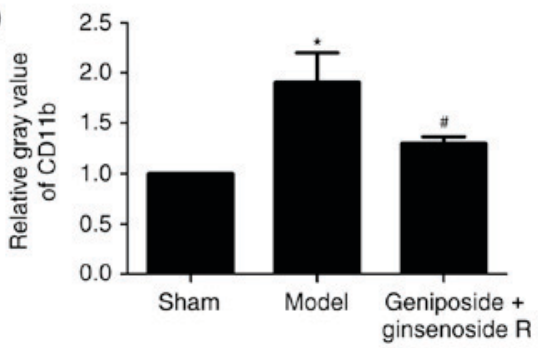

Figure 3. CD11b expression in the brain of middle cerebral artery occlusion model rats with or without geniposide + ginsenoside Rg1 treatment. Sections of brain tissue were incubated with antibody against CD11b for immunohistochemical analysis. (A) A lack of CD11b-antibody labeling was observed in the sham group. (B) Brown staining indicated strong CD11b expression in the model group. (C) Expression of CD11b in the ischemic region of rats was reduced by treatment with geniposide + ginsenoside Rg1, with fewer positively stained cells. Scale bar, $20 \mu \mathrm{m}$. (D) Bar graph indicates the results of the relative gray value obtained by semiquantitative analysis of immunohistochemical staining. ${ }^{\text {" }} \mathrm{P}<0.05$ vs. sham group, ${ }^{\text {" }} \mathrm{P}<0.05$ vs. model group. Geniposide + ginsenoside $\mathrm{R}$, Geniposide + ginsenoside Rg1.

cancer and cardiovascular diseases $(12,13)$. As a critical inflammation-associated miRNA, miR-155 regulates the host immune response by repressing the expression of target genes (38). The majority of target genes of miR-155 are reported to be associated 
A

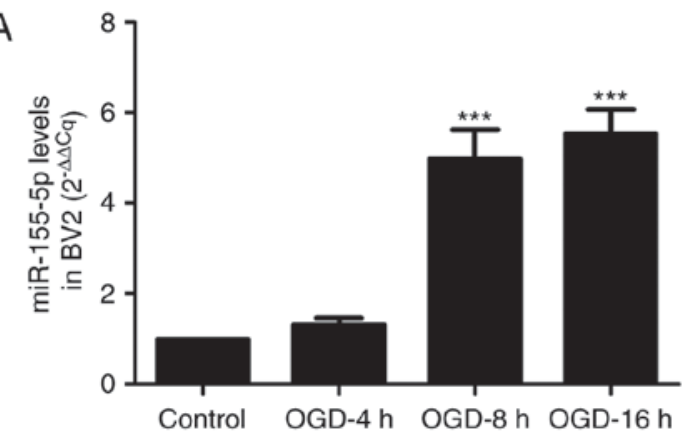

C

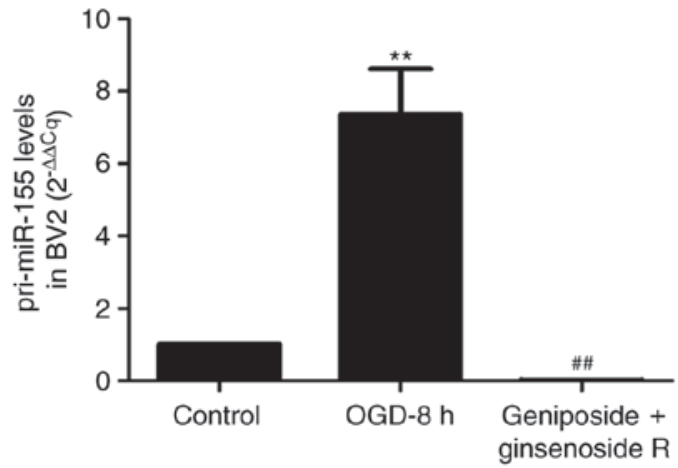

B

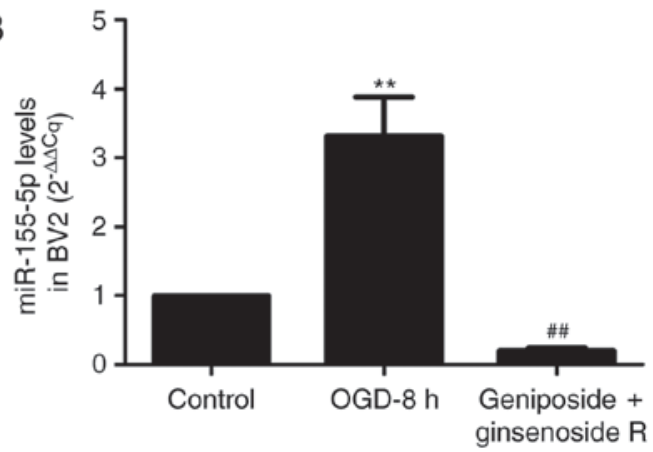

D

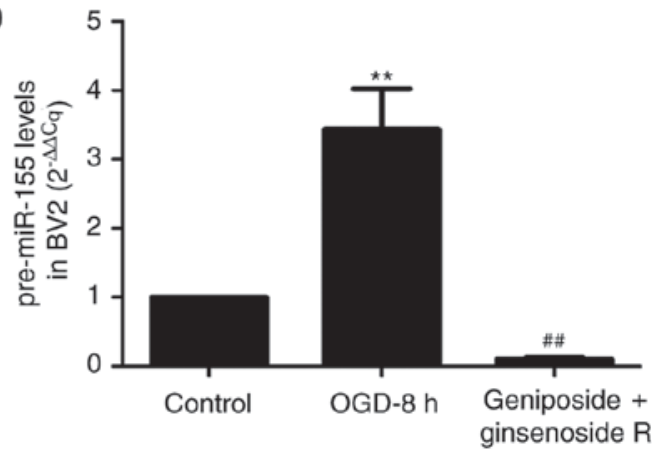

Figure 4. miR-155-5p expression levels in BV2 microglial cells. (A) miR-155-5p expression levels in the microglial cells exposed to OGD injury increased in a time-dependent manner; OGD-4, -8 and $-16 \mathrm{~h}$ denote BV2 microglial cells subjected to OGD for 4, 8 and $16 \mathrm{~h}$, respectively. (B) Geniposide + ginsenoside Rg1 inhibited the expression of mature miR-155-5p in BV2 microglial cells treated with OGD for $8 \mathrm{~h}$. Geniposide + ginsenoside Rg1 suppressed the expression levels of immature precursor (C) pri-miR-155 and (D) pre-miR-155 in BV2 microglial cells treated with OGD for $8 \mathrm{~h}$. Values are presented as the mean + standard deviation, 5 wells per group. ${ }^{* *} \mathrm{P}<0.01$ and ${ }^{* * * *} \mathrm{P}<0.001$ vs. control group; ${ }^{* \#} \mathrm{P}<0.01$ vs. OGD-8 h group. miR, microRNA; OGD, oxygen-glucose deprivation; geniposide + ginsenoside R, geniposide + ginsenoside Rg1.

with the inflammatory regulation process. For example, it has been demonstrated that inflammatory responses mediated by miR-155 in ischemic cerebral tissues were modulated by Toll-like receptor (TLR)4/myeloid differentiation primary response gene 88 and suppressor of cytokine signaling 1 expression, while acetylbritannilactone may suppress miR-155 expression and inhibit inflammatory actions (39). In addition, an association was reported between the expression of TLRs 3, 7 and 9, and miR-155 transcription, in the olfactory bulbs (40). Based on the reported associations between miR-155 and post-ischemic brain inflammation, therapeutic approaches that target miR-155 may be promising for the treatment of cerebral ischemic damage.

Gardenia, the fruit of Gardenia jasminoides Ellis, exhibits varying concentrations of iridoid glycosides. The root of Panax notoginseng (Burk.) F. H. Chen is a widely used traditional Chinese medicine that belongs to the genus Panax and family Araliaceae. Panax notoginseng is also termed Radix notoginseng or Sanchi. In clinical and experimental research, Gardenia is usually used in combination with Panax notoginseng to treat central nervous system diseases, including cerebrovascular disease and Alzheimer's disease. Geniposide and ginsenoside Rg1 are the active components of Gardenia and Panax notoginseng, respectively. In a carrageenan-induced rat paw edema model, Gardenia and Panax notoginseng have been reported to exhibit anti-inflammatory effects via the inhibition of nitric oxide production (41). These compounds were also demonstrated to ameliorate neurological injury, brain infarct volume and the permeability of the blood-brain barrier in MCAO rat models by reducing the expression of proteinase-activated receptor 1 (42). However, studies concerning the role of geniposide + ginsenoside $\mathrm{Rg} 1$ have predominantly been performed in neurons; to the best of our knowledge, investigation regarding their combined effects on microglial cells is limited.

The results of the present study indicated that ischemia caused severe damage in cerebral tissue with a large area of cerebral infarction and brain edema. Geniposide combined with ginsenoside Rg1 reduced the percentage of cerebral infarction size and brain edema in rats subjected to MCAO, which demonstrated a protective effect on ischemia-induced cerebral injury in vivo. RT-qPCR revealed that miR-155 expression levels were unaltered in the 2 and $6 \mathrm{~h}$ ischemia groups; however, when the duration of ischemia was prolonged to $24 \mathrm{~h}$, miR-155 expression levels increased within the brain tissue, compared with the sham group. Notably, miR-155 expression levels were increased in the cortex and basal ganglia in MCAO rats, compared with the sham group, indicating the potential of miR-155 overexpression as a target for the treatment of cerebral ischemic injury. Rats treated with geniposide combined with ginsenoside $\mathrm{Rg} 1$ demonstrated significantly attenuated expression levels of miR-155 expression levels. Based on the results for infarction size and brain edema, the neuroprotective effects of geniposide + ginsenoside Rg1 may be associated with the downregulation of miR-155. In the present study, $10 \%$ chloral hydrate was employed for anesthesia during the course of MCAO surgery, according to the previously described method $(29,30)$ in which all mice or rats were treated with $10 \%$ chloral hydrate for anesthesia; 
however, no symptoms of peritonitis were determined in the present study. In the present study, only the brain tissue was dissected for observation and the abdominal cavity was not observed as there were no obvious symptoms of peritonitis in rats. However, a previous study reported that intraperitoneal administration of $10 \%$ chloral hydrate resulted in adynamic ileus and peritonitis in rats, gastric ulcers in rats and peritonitis in swine (43). Therefore, in future experiments, we plan to change to gas anesthesia with isoflurane.

To the best of our knowledge, activated microglia become hypertrophic, rapidly proliferate and migrate to the inflammatory area where they generate excessive amounts of proinflammatory and neurotoxic cytokines that cause neuronal damage $(44,45)$. In the current study, the results of immunohistochemistry demonstrated that microglial cells increased in number and appeared to possess an activated morphology within ischemic-injured regions. Geniposide + ginsenoside Rg1 markedly reversed the CD11b expression, indicating an inhibitory effect of geniposide + ginsenoside Rg1 on microglial cells. As microglial miR-155 expression serves an important role in the pathological process following ischemic stroke, an in vitro experiment was performed to observe the effects of geniposide + ginsenoside Rg1 on microglial cells. The results demonstrated that miR-155 expression levels were elevated and microglial cells were activated following OGD injury for $8 \mathrm{~h}$, compared with the control group, indicating microglial activation and high inflammatory potential following OGD. However, geniposide + ginsenoside Rg1 reduced the expression of mature miR-155 and the immature precursors, pri-miR-155 and pre-miR-155, indicating a potential protective role of geniposide + ginsenoside $\operatorname{Rg} 1$ in microglial activation. Geniposide + ginsensoside $\operatorname{Rg} 1$ treatment may activate microglial cells to release inflammatory factors. miR-155 is an important miRNA that regulates the mRNA expression of inflammatory factors; based on the inhibitory effect on miR-155 in the current study, the anti-inflammatory effect of geniposide + ginsenoside Rg1 may be achieved by inhibiting miR-155 expression.

In conclusion, the in vivo and in vitro experiments of the present study demonstrated that the administration of geniposide in combination with ginsenoside Rg1 via tail vein injection inhibited ischemia-induced microglial cell activation, which may be associated with the inhibition of microglial miR-155-5p expression. The results of the present study indicated that geniposide in combination with ginsenoside Rg1 may serve as a potential alternative for the prevention of microglial cell injury in ischemic diseases.

\section{Acknowledgements}

The present study was supported by the Natural Science Foundation in China (grant no. 81473449).

\section{References}

1. Xu F,Li J,Ni W, Shen YW andZhang XP: Peroxisome proliferatoractivated receptor-gamma agonist $15 \mathrm{~d}$-prostaglandin $\mathrm{J} 2$ mediates neuronal autophagy after cerebral ischemia-reperfusion injury. PLoS One 8: e55080, 2013.

2. Cheng CY and Lee YC: Anti-Inflammatory effects of traditional chinese medicines against ischemic injury in in vivo models of cerebral ischemia. Evidence Based Complement Alternat Med 2016: 5739434, 2016.
3. Liu X, Tao Y, Wang F, Yao T, Fu C, Zheng H, Yan Y, Liang X, Jiang $X$ and $Z$ hang $Y$ : Kudiezi injection mitigates myocardial injury induced by acute cerebral ischemia in rats. BMC Complement Alternat Med 17: 8, 2017.

4. Ambros V: The functions of animal microRNAs. Nature 431: 350-355, 2004.

5. Bartel DP: MicroRNAs: Target recognition and regulatory functions. Cell 136: 215-233, 2009.

6. Lau P and Hudson LD: MicroRNAs in neural cell differentiation. Brain Res 1338: 14-19, 2010.

7. Rybak A, Fuchs H, Smirnova L, Brandt C, Pohl EE, Nitsch R and Wulczyn FG: A feedback loop comprising lin-28 and let-7 controls pre-let-7 maturation during neural stem-cell commitment. Nat Cell Biol 10: 987-993, 2008.

8. White RE and Giffard RG: MicroRNA-320 induces neurite outgrowth by targeting ARPP-19. Neuroreport 23: 590-595, 2012.

9. Lim KY, Chua JH, Tan JR, Swaminathan P, Sepramaniam S, Armugam A, Wong PT and Jeyaseelan K: MicroRNAs in Cerebral Ischemia. Transl Stroke Res 1: 287-303, 2010.

10. Liu DZ, Tian Y, Ander BP, Xu H, Stamova BS, Zhan X, Turner RJ, Jickling G and Sharp FR: Brain and blood microRNA expression profiling of ischemic stroke, intracerebral hemorrhage, and kainate seizures. J Cereb Blood Flow Metab 30: 92-101, 2010.

11. Liu Y, Zhang J, Han R, Liu H, Sun D and Liu X: Downregulation of serum brain specific microRNA is associated with inflammation and infarct volume in acute ischemic stroke. J Clin Neurosci 22: 291-295, 2015.

12. Faraoni I, Antonetti FR, Cardone J and Bonmassar E: miR-155 gene: A typical multifunctional microRNA. Biochim Biophys Acta 1792: 497-505, 2009.

13. O'Connell RM, Kahn D, Gibson WS, Round JL, Scholz RL, Chaudhuri AA, Kahn ME, Rao DS and Baltimore D: MicroRNA-155 promotes autoimmune inflammation by enhancing inflammatory $\mathrm{T}$ cell development. Immunity 33: 607-619, 2010.

14. Dheen ST, Kaur C and Ling EA: Microglial activation and its implications in the brain diseases. Curr Med Chem 14: 1189-1197, 2007.

15. Butovsky O, Jedrychowski MP, Cialic R, Krasemann S, Murugaiyan G, Fanek Z, Greco DJ, Wu PM, Doykan CE, Kiner O, et al: Targeting miR-155 restores abnormal microglia and attenuates disease in SOD1 mice. Ann Neurol 77: 75-99, 2015.

16. Cardoso AL, Guedes JR, Pereira de Almeida L and Pedroso de Lima MC: miR-155 modulates microglia-mediated immune response by down-regulating SOCS-1 and promoting cytokine and nitric oxide production. Immunology 135: 73-88, 2012.

17. Pareek S, Roy S, Kumari B, Jain P, Banerjee A and Vrati S: MiR-155 induction in microglial cells suppresses Japanese encephalitis virus replication and negatively modulates innate immune responses. J Neuroinflammation 11: 97, 2014.

18. Chen QC, Zhang WY, Kim H, Lee IS, Ding Y, Youn UJ, Lee SM, Na M, Min BS and Bae K: Effects of Gardeniae Fructus extract and geniposide on promoting ligament cell proliferation and collagen synthesis. Phytother Res 24 (Suppl 1): S1-S5, 2010.

19. Wang J, Li PT, Du H, Hou JC, Li WH, Pan YS and Chen HC: Tong Luo Jiu Nao injection, a traditional Chinese medicinal preparation, inhibits MIP- $1 \beta$ expression in brain microvascular endothelial cells injured by oxygen-glucose deprivation. J Ethnopharmacol 141: 151-157, 2012.

20. Wang SW, Lai CY and Wang CJ: Inhibitory effect of geniposide on aflatoxin B1-induced DNA repair synthesis in primary cultured rat hepatocytes. Cancer Lett 65: 133-137, 1992.

21. Lee P, Lee J, Choi SY, Lee SE, Lee S and Son D: Geniposide from Gardenia jasminoides attenuates neuronal cell death in oxygen and glucose deprivation-exposed rat hippocampal slice culture. Biol Pharm Bull 29: 174-176, 2006.

22. Wang J, Hou J, Zhang P, Li D, Zhang C and Liu J: Geniposide reduces inflammatory responses of oxygen-glucose deprived rat microglial cells via inhibition of the TLR4 signaling pathway. Neurochem Res 37: 2235-2248, 2012.

23. Zhang Y, Lin SX, Hua Q, Yang KY, Yao N, Yi LS, Wang AM, Chen WJ and Chen JY: Research on the mechanism of the active ingredients of Sanchi and Gardenia removing the early amyloid in the AD transgenic mouse brain. Chin Pharmacol Bull 28: 179-84, 2012 (In Chinese).

24. Zhai YS, Du SY, Lu Y, Wang Y, Xu B and Gao Y: Impact of notoginseng total saponin on intestinal absorption kinetics of jasminoidin. China J Trad Chin Med Pharm 25: $459-462,2010$ 
25. Lanou W, Heqin Z, Wenjun $\mathrm{H}$ and Wenfeng C: Analysis of behavioral index of ginsenoside_RG 1 intervention on brain ischemia. Chin J Lab Ani Sci 14: 283-285, 2004.

26. Xia L, Cuifen B, Jia W, Jia L and Shujian Q: The protective role and mechanism of Ginsenoside Rg1 on the ischemic-reperfusion injuried neurons in hippocampus CA1 of rat. Progress Anat Sci 16: 177-180, 2010.

27. Hollands C: The animals (scientific procedures) act 1986. Lancet 2: 32-33, 1986.

28. Longa EZ, Weinstein PR, Carlson S and Cummins R: Reversible middle cerebral artery occlusion without craniectomy in rats. Stroke 20: 84-91, 1989.

29. Liu JH, Feng D, Zhang YF, Shang Y, Wu Y, Li XF and Pei L: Chloral hydrate preconditioning protects against ischemic stroke via upregulating annexin A1. CNS Neuroscience Ther 21: 718-726, 2015

30. Chang CF, Lin SZ, Chiang YH, Morales M, Chou J, Lein P, Chen HL, Hoffer BJ and Wang Y: Intravenous administration of bone morphogenetic protein-7 after ischemia improves motor function in stroke rats. Stroke 3: 558-564, 2003.

31. Livak KJ and Schmittgen TD: Analysis of relative gene expression data using real-time quantitative PCR and the 2(-Delta Delta C(T)) method. Methods 25: 402-408, 2001.

32. Brouns R and De Deyn PP: The complexity of neurobiological processes in acute ischemic stroke. Clin Neurol Neurosurg 111: 483-495, 2009.

33. Liguz-Lecznar M and Kossut M: Influence of inflammation on poststroke plasticity. Neural Plast 2013: 258582, 2013

34. Taganov KD, Boldin MP and Baltimore D: MicroRNAs and immunity: Tiny players in a big field. Immunity 26: 133-137, 2007.

35. O'Connell RM, Rao DS, Chaudhuri AA and Baltimore D Physiological and pathological roles for microRNAs in the immune system. Nat Rev Immunol 10: 111-122, 2010.

36. Xiao $\mathrm{C}$ and Rajewsky $\mathrm{K}$ : MicroRNA control in the immune system: Basic principles. Cell 136: 26-36, 2009.
37. Zhu J, Yue H, Qiao C and Li Y: Association between single-nucleotide polymorphism (SNP) in miR-146a, miR-196a2, and miR-499 and risk of ischemic stroke: A meta-analysis. Med Sci Monit 21: 3658-3663, 2015

38. Wang J, Yang K, Zhou L, Minhaowu, Wu Y, Zhu M, Lai X, Chen T, Feng L, Li M, et al: MicroRNA-155 promotes autophagy to eliminate intracellular mycobacteria by targeting Rheb. PLoS Pathog 9: e1003697, 2013.

39. Wen Y, Zhang X, Dong L, Zhao J, Zhang C and Zhu C: Acetylbritannilactone modulates MicroRNA-155-mediated inflammatory response in ischemic cerebral tissues. Mol Med 21: 197-209, 2015.

40. Oliveira BRSM, Vieira FV, de S Vieira D, da Silva SEL, Gameiro R, Flores EF and Cardoso TC: Expression of miR-155 associated with Toll-like receptors 3,7 , and 9 transcription in the olfactory bulbs of cattle naturally infected with BHV5. J Neurovirol 23: 772-778, 2017.

41. Zheng X, Yang D, Liu X, Wang N, Li B, Cao H, Lu Y, Wei G, Zhou $\mathrm{H}$ and Zheng J: Identification of a new anti-LPS agent, geniposide, from Gardenia jasminoides Ellis, and its ability of direct binding and neutralization of lipopolysaccharide in vitro and in vivo. Int Immunopharmacol 10: 1209-1219, 2010.

42. Xie CL, Li JH, Wang WW, Zheng GQ and Wang LX Neuroprotective effect of ginsenoside-Rg1 on cerebral ischemia/reperfusion injury in rats by downregulating protease-activated receptor-1 expression. Life Sci 121: 145-151, 2015.

43. Silverman J and Muir WW III: A review of laboratory animal anesthesia with chloral hydrate and chloralose. Lab Anim Sci 43: 210-216, 1993.

44. Smith JA, Das A, Ray SK and Banik NL: Role of pro-inflammatory cytokines released from microglia in neurodegenerative diseases. Brain Res Bull 87: 10-20, 2012.

45. Vilhardt F: Microglia: Phagocyte and glia cell. Int J Biochem Cell Biol 37: 17-21, 2005 